Recepción: 19 / 02 / 2019

Aceptación: 18 / 03 / 2019

Publicación: 05 / 06 / 2019
Ciencias técnicas y aplicadas

Artículo de investigación

\title{
Comunicación parcial neurosensorial-visual a un ordenador mediante lectura de pupila
}

\section{Partial neurosensory-visual communication to a computer through pupil reading}

\section{Comunicação neurosensorial-visual parcial a um computador através da leitura de alunos}

Mónica M. Miranda-Ramos I mmiranda@ups.edu.ec

Jaime J. Moscoso-Cabrera II lmontesdeoca@est.ups.edu.ec

Luiggy A. Montesdeoca-Jácome ${ }^{\text {III }}$ jmoscosoc@est.ups.edu.ec

Correspondencia: mmiranda@ups.edu.ec

\footnotetext{
I. Magíster en Automatización y Control Industrial, Docente Universidad Politécnica Salesiana, Quito, Ecuador.

II. Estudiante Ingeniería Electrónica Especialización Automatización Industrial, Universidad Politécnica Salesiana, Quito, Ecuador.

III. Estudiante Ingeniería Electrónica Especialización Automatización Industrial, Universidad Politécnica Salesiana, Quito, Ecuador.
} 


\title{
Resumen
}

La mayoría de personas con discapacidad motora presentan inconvenientes en el desarrollo de sus actividades diarias, tanto en el ámbito personal como profesional. Para contribuir con una mejor calidad de vida, se presentó el proyecto de investigación basado en componentes electrónicos, cuyo objetivo fue verificar la comunicación parcial neurosensorial-visual a un ordenador mediante la lectura de pupila. La metodología empleada se sustenta en el desarrollo de un sistema para el control del computador mediante la detección de rostros, centrándonos en la zona de los ojos y específicamente en el seguimiento de la pupila para el control del movimiento del cursor del mismo. Mediante electroencefalografía (EEG) se registra y utiliza principalmente la onda cerebral Alfa para realizar las operaciones de los clics en el ordenador mediante parpadeos voluntarios, el usuario efectuará dos o tres parpadeos para dar la orden al equipo de realizar un clic izquierdo o clic derecho respectivamente. Como dispositivo para la captación de rostro y movimiento de la pupila se usa una cámara web y en lo que respecta a la de adquisición de datos (ondas cerebrales) se utiliza una diadema MindWave ${ }^{\mathrm{TM}}$ Mobile 2 (Interfaz cerebro-computadora) la cual mide la actividad eléctrica del cerebro. Todo el proceso, datos y acciones respectivas a ejecutar son controlados mediante el software Matlab. Se obtuvo un sistema de Comunicación Neurosensorial-Visual, que fue valorado por usuarios con discapacidad y expertos en informática, quienes determinaron en promedio, un nivel de "muy satisfactorio", a partir de una encuesta. Se concluyó que existen señales bioeléctricas cerebrales susceptibles de ser detectadas y traducidas por dispositivos electrónicos. Mediante algoritmos se pueden convertir estas señales en actividades sustitutas para el individuo como el movimiento del cursor de un computador a través de la pupila. El sistema evidenció su funcionamiento adecuado y utilidad infinita.

Palabras claves: Control; detección; electroencefalografía (EEG); pupila, parpadeo; interfaz cerebrocomputadora (BCI), Mindwave Mobile 2; Matlab.

\begin{abstract}
The majority of people with motor disabilities have problems in the development of their daily activities, both in the personal and professional fields. To contribute to a better quality of life, we presented the research project based on electronic components, whose objective was to verify the partial neurosensory-visual communication to a computer through pupil reading. The methodology
\end{abstract}


used is based on the development of a system for the control of the computer by the detection of faces, focusing on the area of the eyes and specifically on the follow-up of the pupil for the control of the movement of the cursor. By means of electroencephalography (EEG) the Alpha brainwave is registered and used mainly to perform click operations on the computer through voluntary blinks, the user will make two or three blinks to give the order to the device to make a left click or right click respectively. A webcam is used as a device for the capture of the face and movement of the pupil and a MindWave TM Mobile 2 headset (brain-computer interface) is used in terms of data acquisition (brainwaves), which measures the electrical activity of the Brain. All the process, data and respective actions to be executed are controlled by the Matlab software. A neurosensory-Visual communication system was obtained, which was valued by disabled users and computer experts, who determined on average a "very satisfactory" level, from a survey. It was concluded that there are bioelectrical brain signals susceptible to be detected and translated by electronic devices. By means of algorithms these signals can be converted into surrogate activities for the individual as the movement of the cursor of a computer through the pupil. The system demonstrated its proper functioning and infinite utility.

Keys words: Control, detection, electroencephalography (EEG), pupil, blink, brain-computer Interface (BCI), Mindwave Mobile 2, Matlab

\section{Resumo.}

A maioria das pessoas com deficiência motora tem problemas no desenvolvimento de suas atividades diárias, tanto no campo pessoal como no profissional. Para contribuir para uma melhor qualidade de vida, apresentamos o projeto de pesquisa baseado em componentes eletrônicos, cujo objetivo foi verificar a comunicação parcial neurossensorial-visual a um computador através da leitura de alunos. A metodologia utilizada baseia-se no desenvolvimento de um sistema para o controle do computador pela detecção de rostos, com foco na área dos olhos e, especificamente, no acompanhamento do aluno para o controle do movimento do cursor. Por meio de eletroencefalografia (EEG), a onda cerebral Alpha é registrada e usada principalmente para realizar operações de cliques no computador através de piscadas voluntárias, o usuário fará duas ou três piscadas para dar a ordem ao dispositivo para clicar com o botão esquerdo ou direito, respectivamente. Uma webcam é usada como um dispositivo para a captura do rosto e do movimento do aluno e um fone de ouvido MindWave ${ }^{\text {TM }}$ Mobile 2 (interface cérebro-computador) é usado em termos de aquisição de dados (ondas cerebrais), que mede a atividade elétrica do cérebro . Todo o processo, dados e respectivas ações a serem executadas são controlados 
pelo software Matlab. Foi obtido um sistema de comunicação neurossensorial-visual, valorizado por usuários com deficiência e especialistas em informática, que determinaram em média um nível "muito satisfatório", a partir de uma pesquisa. Concluiu-se que existem sinais cerebrais bioelétricos suscetíveis de serem detectados e traduzidos por dispositivos eletrônicos. Por meio de algoritmos, esses sinais podem ser convertidos em atividades substitutas para o indivíduo, como o movimento do cursor de um computador através da pupila. O sistema demonstrou seu funcionamento adequado e utilidade infinita.

Palavras chaves: Controle, detecção, eletroencefalografia (EEG), pupila, piscar, interface cérebrocomputador (BCI), Mindwave Mobile 2, Matlab.

\section{Introducción.}

La Organización Mundial de la Salud define como discapacidad a la restricción o ausencia de capacidad para realizar una actividad normal para el ser humano, la misma que puede ser física, psicosocial, cognitiva o sensorial, éstas se pueden producir por factores infecciosos, neurológicos, reumáticos, musculares o traumáticos (Giaconi, Pedrero, \& San Martín, 2017). Una persona con una afección del aparato locomotor más o menos grave y de larga evolución, se considera que tiene discapacidad motora, la cual limita sus actividades, pues presenta dificultades para caminar y manipular los objetos de su entorno como por ejemplo el ratón de la computadora (M. Martín \& Sánchez, 2016). En el mundo moderno la interacción con la tecnología constituye la base del desarrollo de los pueblos y quedarse a la saga de este recurso limita a esta población discapacitada aún más.

La información que da el Consejo Nacional para la Igualdad de Discapacidades (CONADIS) en el Ecuador manifiesta que existe un $46.68 \%$ de personas con algún tipo de discapacidad física y en la provincia del Guayas específicamente en la ciudad de Guayaquil un 46.99\% también la tiene (CONADIS, s. f.).

El ojo humano es capaz de transformar el fotón en un impulso eléctrico mediante las células foto receptoras que posee en su retina, dicho impulso puede ser procesado por el cerebro en el campo visual (Puentes, Puentes, Puentes, \& Chávez, 2017), estos impulsos pueden ser utilizados para órdenes de 
control y manipular dispositivos externos como ordenadores de tal forma que existe una gran posibilidad de lograr la conexión entre una persona con discapacidad motora y la tecnología.

En la actualidad se conoce que se puede registrar la actividad neuronal y los movimientos de la pupila mediante la transmisión de señales eléctricas, las mismas que pueden ser tratadas con nuevas tecnologías tales como el sistema "Interfaz cerebro - computadora", que se basa en la adquisición de ondas cerebrales para luego ser procesadas e interpretadas por un ordenador (Rodríguez \& Duque, 2016) (J. Martín, 2015) (Pérez, 2017). Este recurso puede solucionar el problema de conectividad de las personas con discapacidad física.

Mediante las interfaces cerebro computadora (BCI, Brain Computer Interfaces) que son dispositivos que captan las señales eléctricas de la corteza cerebral de una persona, para posteriormente enviarlas a una computadora para su despliegue o su procesamiento, puede existir una comunicación entre el ser humano y la tecnología, hasta el punto de manipular dispositivos del medio físico como ordenadores, coches de juguete e incluso sillas de ruedas, sin la necesidad de usar las extremidades y sólo usando nuestro cerebro (Alvarado, Tinoco, \& Veintimilla, 2016) (Morín, Santillán, Sainos, \& Oliveros, 2018) (L. Medina, 2018). Por lo que éste trabajo ha sido motivado para proporcionar una ayuda significativa en el desarrollo intelectual de las personas con discapacidad, dándoles la oportunidad de utilizar su computador para el desarrollo cognitivo, personal e incluso profesional.

La presente investigación tiene como objetivo verificar la comunicación parcial neurosensorial-visual a un ordenador mediante lectura de pupila. Los objetivos específicos van orientados a: adquirir señales bioeléctricas del usuario, establecer el proceso de sinapsis neuronal, desarrollar algoritmos para el proceso de adquisición y transducción de señales electroencefalográficas y pupilares con sus respectivas simulaciones, identificar las limitantes del entorno y evaluar el prototipo.

\section{Metodología.}

Se trata de un proyecto de diseño experimental, metodología descriptiva y analítica, para lo cual se propone un algoritmo que permita la comunicación entre un individuo con discapacidad motriz y el ordenador a través del rastreo ocular.

Para el desarrollo de ésta investigación se ha necesitado una diadema que registra la actividad cerebral y una cámara web para el rastreo ocular, además se ha implementado el algoritmo de control que 
interactúa entre los elementos que permiten la manipulación del computador y las acciones que se llevan a cabo en el mismo. Todo éste conjunto conforma el prototipo denominado "CONEVI" (Comunicación Neurosensorial-Visual).

\section{Materiales}

Para la realización de este proyecto se utilizó una computadora portátil con sistema operativo Windows 10 la cual trabaja con un procesador Intel Core i5 trabajando a $1.8 \mathrm{GHz}$ con 12 GB de memoria RAM, a la cual se le acopló una cámara web de buen desempeño con una resolución máxima de 1280 x 720 p HD, un dispositivo o diadema que recoge la actividad cerebral capaz de medir, principalmente, los estados de relajación y atención. Todo esto a través de los ritmos cerebrales: alfa, beta, gamma; también es capaz de captar el parpadeo. Adicionalmente, para la implementación del algoritmo que realiza todo el procesamiento de la imagen obtenida de la cámara web y procesamiento de las señales neuronales se utilizó el programa MATLAB que es un entorno de computación y desarrollo de aplicaciones totalmente integrado que dispone de un amplio abanico de programas de apoyo denominados 'Toolboxes' entre los que destacan el 'toolbox' de proceso de imágenes, control robusto, matemáticas simbólicas, redes neurales, etc. Con los que se pueden ampliar las capacidades del software (Chang, 2014).

\section{Procedimiento}

Se propuso un algoritmo capaz de permitir la conexión del computador con una cámara web y un dispositivo que adquiere las ondas cerebrales. Estos algoritmos se desarrollaron en el software de programación Matlab y fueron sometidos a simulación para evidenciar su correcto funcionamiento. Mediante la cámara web, se procedió al reconocimiento de rostro y captura del movimiento de la pupila del usuario, para permitirle la manipulación del cursor del computador, para que el usuario observe y dirija de manera correcta el movimiento del cursor en la pantalla del mismo, este dispondrá de una interfaz gráfica de usuario (Ferreira \& Rodríguez, 2017) en la que se encuentra un recorte de la imagen de su rostro, recorte de la imagen de su ojo, gráfica de la señal Alfa y un cuadro de diálogo que indica al usuario que vea fijamente el cursor para que el programa funcione de manera óptima. Con el dispositivo "Mindwave Mobile 2" de "NeuroSky" el usuario da la orden al equipo de realizar un clic izquierdo (dos parpadeos) o un clic derecho (tres parpadeos). En la Figura 1 se pueden observar los pasos seguidos para cumplir con cada proceso mencionado. 




Figura 1. Diagrama de bloques para el control del cursor y clics con "CONEVI".

Fuente: (Autores, 2019)

En esta propuesta la toma de señal e imagen, su procesamiento en MATLAB y la ejecución de las acciones respectivas de control del computador se llevan a cabo mediante renombramientos de datos obtenidos sobre una misma variable simulando el tiempo real. Se presenta el sistema propuesto interfaz cerebro computadora (BCI) por sus siglas en inglés, en la Figura 2.

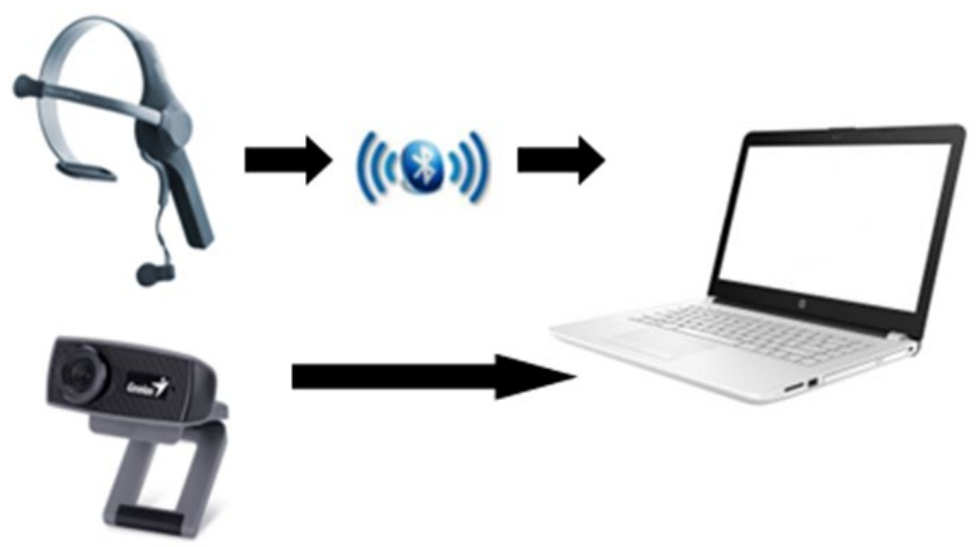

Figura 2. Diagrama de Bloques del BCI Propuesto

Fuente: (Autores, 2019)

Mindwave Mobile 2 
Los sistemas de adquisición de señales de EEG son complejos, entre el sistema de hardware y software tienen una encriptación lo cual impide un post procesamiento de la bioseñal para tareas específicas, además del hecho de que ya existen dispositivos comerciales para dicha tarea, por lo que se optó por el uso de un sistema BCI (Interfaz Cerebro-Computadora) tipo diadema del fabricante "NeuroSky", denominado "Mindwave Mobile 2", el mismo que se aprecia en la Figura 3.

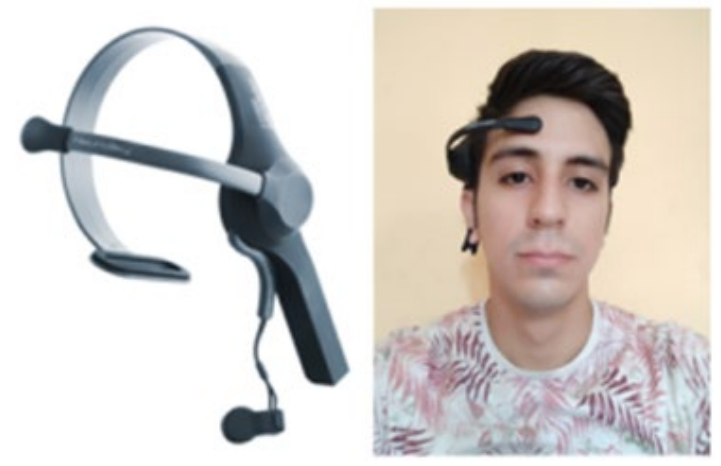

Figura 3. Dispositivo MindWave Mobile 2.

Fuente: (Autores, 2019)

Dicha diadema capta la señal de EEG pero debido a que posee una magnitud muy pequeña (mili voltios), tiene que ser amplificada, luego es filtrada para eliminar cualquier tipo de ruido y finalmente es digitalizada para ser convertida en comandos que el computador entiende y que el usuario desea controlar, con dichos comandos se pueden efectuar las acciones correspondientes en el dispositivo final que en este caso es el computador (Altamirano \& Revilla, 2017). En la Figura 4 se pude apreciar el proceso BCI.

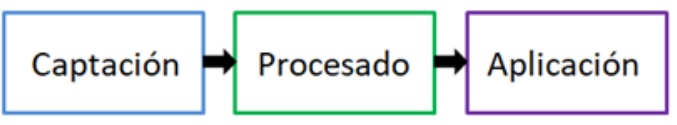

Figura 4. Diagrama de Bloques Proceso BCI.

Fuente: (Autores, 2019)

El proceso de captación de la señal se logra gracias a un electrodo que se coloca en la frente en la posición de Fp1 del sistema médico 10-20 (Moctezuma, Carrillo, Villaseñor, \& Torres, 2017) y un electrodo de referencia en el lóbulo de la oreja. 


\section{Cámara web}

La cámara web debe estar ubicada correctamente sobre el computador de manera que capte de forma precisa la zona de interés, es decir, el rostro del usuario, para posteriormente centrase en la detección de la zona de los ojos y realizar el corte de uno solo (ojo lado derecho), como valor agregado, a la cámara se añadió visión nocturna, se construyó un aro provisto por leds infrarrojos los cuáles son alimentados por una batería de 9 Voltios y protegidos por una resistencia añadida al cable de alimentación para que los leds no se quemen (Figura 5). Cabe recalcar que comúnmente la mayoría de cámaras vienen incorporadas con un filtro IR que impide ver el espectro de luz infrarroja, por lo que si es necesario hay que abrir la cámara y retirar el mismo.

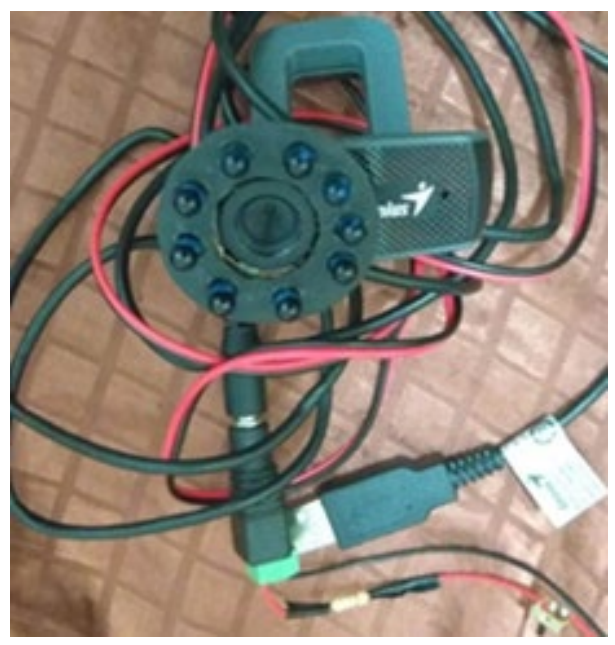

Figura 5. Cámara Web con Aro de LEDs Infrarrojos.

Fuente: (Autores, 2019)

\section{Control del Cursor por Movimientos Oculares}

Para el control del cursor del computador utilizando los movimientos oculares se obtiene la imagen del usuario mediante la cámara web, la misma que debe apuntar siempre al rostro del usuario para tener una buena captación facial, la imagen obtenida inicialmente está en formato RGB (imagen a color) y para un procesamiento óptimo es posteriormente transformada a escala de grises, luego se gira la imagen de izquierda a derecha para generar un efecto espejo, se binariza y se rellenan las áreas blancas existentes dentro de la vista para una mayor detección, se delimita la zona de la imagen, conocida como región de interés ROI (Region Of Interest, en inglés) (Justo \& Aguirre, 2014), que en 
este caso es la zona del rostro, el cual está localizado en un cuadro amarillo y para mayor exactitud centramos la detección en la zona de los ojos encerrados en un cuadro de color azul como se puede apreciar en la Figura 6. Para la detección se estudió y se tuvo como guía el método de clasificadores de cascada, el cual busca características tipo Haar de una estructura en específico dentro de una imagen, este método fue propuesto por Viola-Jones y adaptado a nuestras necesidades (Rodríguez \& Duque, 2016).

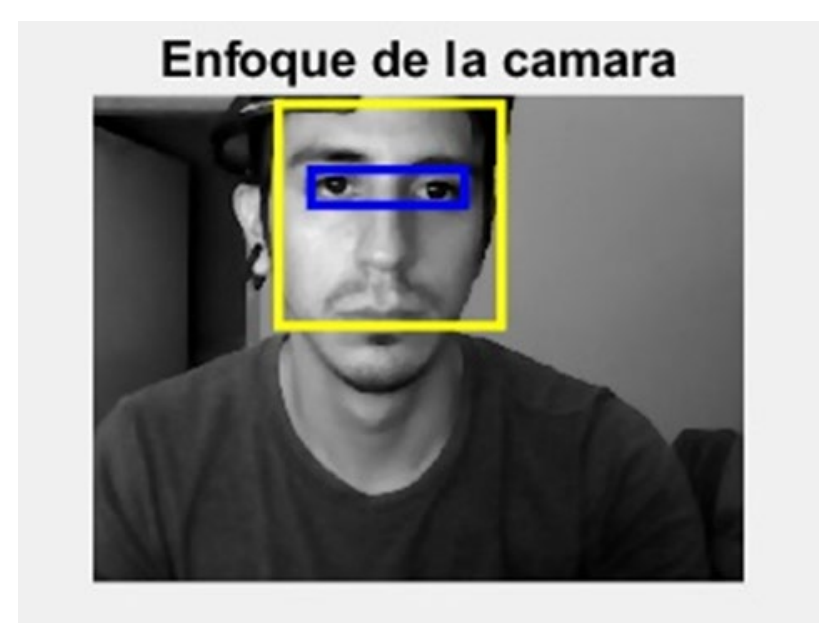

Figura 6. Detección de rostro y ojos.

Fuente: (Autores, 2019)

\begin{abstract}
Al identificar la sección de la vista se hace un recorte de la misma, específicamente del ojo derecho y mediante un filtro en el que se puede variar numerador y denominador se convierten los pixeles negativos en positivos disminuyendo o eliminando cualquier brillo excedente en los ojos provocado por algún resplandor o reflejo, con la imagen adecuada se procede a la identificación de la pupila localizándola mediante un círculo de color verde ajustable al tamaño de la misma, adicionalmente se agregó una cruz color turquesa que simula el cursor y un rectángulo de color rojo que representa de manera escalada la pantalla del computador, como se puede observar en la Figura 7. De esta forma el usuario puede conocer la posición del mismo y ubicarlo en donde desee.
\end{abstract}




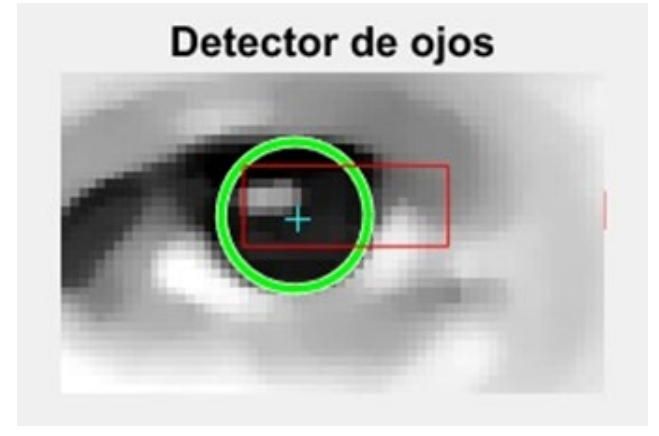

Figura 7. Cruz turquesa (cursor), Rectángulo rojo (pantalla).

Fuente: (Autores, 2019)

\section{Control para la Acción de Clics}

Los impulsos eléctricos que produce nuestro cerebro son información que viaja de neurona a neurona haciendo uso de cientos de miles de ellas para conseguir transportarse y efectuar una función determinada. Estos impulsos producen ritmos llamados ondas cerebrales, existen cuatro tipos principales que son: alfa, beta, theta y delta (Altamirano \& Revilla, 2017), las cuales se observan en la Figura 8.

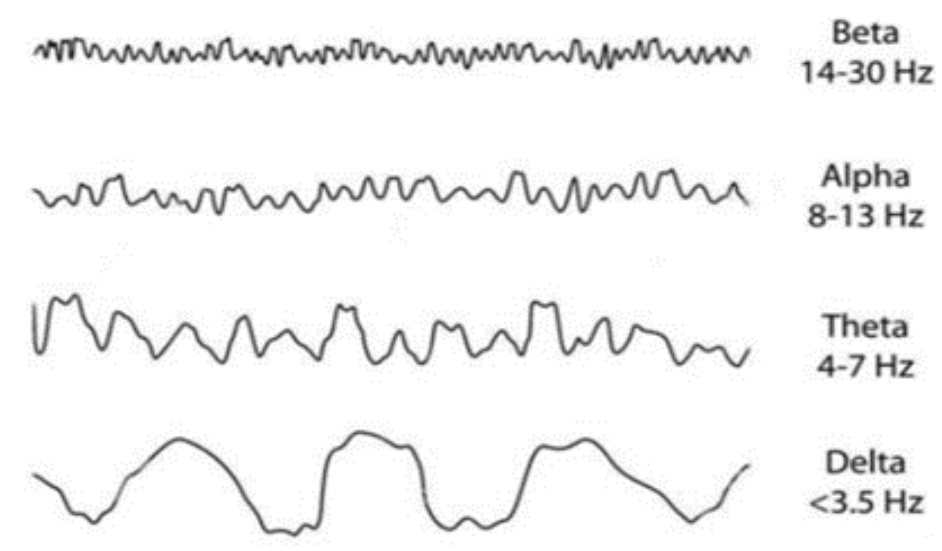

Figura 8. Ondas Cerebrales y sus Frecuencias.

Fuente: (Iglesias López, 2016)

Para el control de clics se toman las señales que entrega la diadema Mindwave Mobile 2, hay señales que son afectadas por estímulos específicos, la señal Alfa está relacionada con la corteza visual y existe variación en ella con algún tipo de impulso ocular (Sánchez, Jofré, \& Burán, 2018), con el 
parpadeo se identificó una clara y considerable alteración en la misma, por eso fue utilizada como base para realizar el control en cuanto a los clics del computador, cuando el usuario cierra los ojos existe un pico positivo o negativo en la señal que indica que ha realizado un parpadeo tal como se puede observar en la Figura 9.

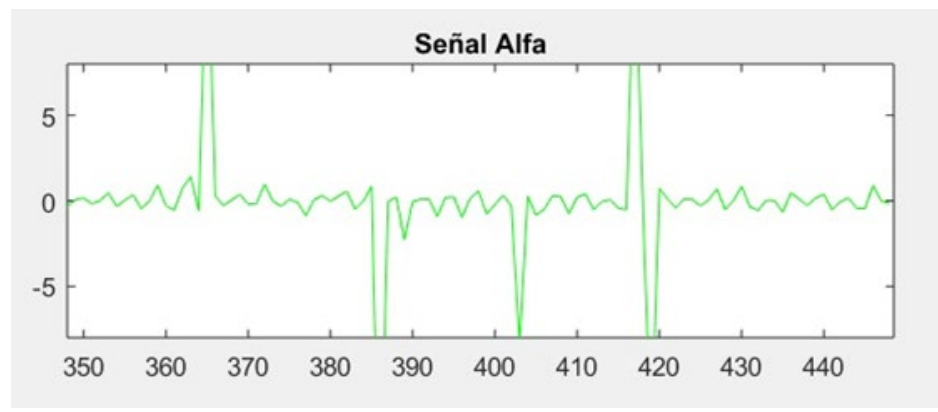

Figura 9. Picos en la Señal al Parpadear.

Fuente: (Autores, 2019)

Ya que realizamos parpadeos involuntarios y no deseamos que estos sean considerados como clics, después de que el usuario da un primer parpadeo se considera un tiempo de 5 segundos para que dentro de este tiempo pueda realizar más parpadeos voluntarios considerándose de esta manera, 2 parpadeos como un clic izquierdo y 3 parpadeos como un clic derecho. En la Figura 10. se puede observar como la señal sufre 2 picos cuando se ha realizado un clic izquierdo y 3 picos cuando se efectúa un clic derecho.



Figura 10. Clic izquierdo y clic derecho visualizados en la señal.

Fuente: (Autores, 2019) 
Una vez concluida esta fase del proyecto se procedió a validar el funcionamiento de todo el sistema “CONEVI” para lo cual se seleccionó una muestra intencional constituida por expertos en informática, individuos con discapacidad motora parcial o total e individuos con alguna morbilidad. Los participantes firmaron un consentimiento informado y contribuyeron con la realización de una encuesta basada en la escala de Likert que asignaba una puntuación entre 1 y 5 , correspondiente a insatisfactorio, poco satisfactorio, medianamente satisfactorio, satisfactorio y muy satisfactorio, respectivamente. Esta encuesta permite evidenciar el grado de aceptación con el funcionamiento del “CONEVI” y además la sumatoria caracteriza el nivel de satisfacción del usuario.

Las preguntas que contestaron los usuarios fueron:

1.- Las instrucciones de uso impartidas son fáciles de seguir.

2.- El movimiento del cursor del computador estuvo acorde al movimiento de la pupila.

3.- La respuesta en el ordenador es adecuada (clic izquierdo-derecho).

4.- La utilización de "CONEVI” es segura (libre de incidentes).

5.- La comunicación humano-computador es factible mediante "CONEVI".

Basados en la encuesta, los autores utilizando la sumatoria del puntaje obtenido, interpretaron el nivel de satisfacción de los usuarios de acuerdo a la siguiente ponderación:

\section{5 -25: Muy Satisfactorio}

17.5 - 22.4: Satisfactorio

12.5 - 17.4: Medianamente Satisfactorio

7.5 - 12.4: Poco Satisfactorio

$<7.5$ : Insatisfactorio

\section{Resultados.}


Se obtuvieron como resultados de esta investigación el algoritmo con funcionalidad completa en todas las fases del desarrollo, desde el diseño hasta la prueba de evaluación realizada por los usuarios.

Otros resultados obtenidos derivan de la aplicación del proyecto en una muestra intencionada constituida por 30 participantes entre los cuales se contó con 5 profesionales, 7 personas con morbilidad y 8 personas con discapacidad física.

Tabla 1. Medidas de tendencia central

\begin{tabular}{|l|l|l|}
\hline MEDIDAS & EDAD_AÑOS & $\begin{array}{l}\text { VALORACIÓN_CONEV } \\
\text { I /25 PUNTOS }\end{array}$ \\
\hline Media & 44 & 23 \\
Mediana & 44 & 24 \\
Moda & 58 & 25 \\
Desviación estándar & 13 & 2 \\
Mínimo & 19 & 20 \\
Máximo & 60 & 25 \\
\hline
\end{tabular}

Fuente: Base de datos

\section{Elaborado por: Autores}

Interpretación. Se puede observar que la media de edad en la muestra de estudio es de 44 años, con un mínimo de 19 años y un máximo de 60. La escala de valoración del proyecto denominado "CONEVI" demuestra un alto nivel de aceptación, ya que la media fue de 23 puntos sobre un máximo de 25 .

\section{DATOS DEMOGRÁFICOS}


Gráfico 1. Datos Demográficos de la muestra

Fuente: Base de datos.

Elaborado por: Autores

Interpretación. Los resultados del gráfico 1 evidencian que el grupo etario que predominó en la muestra fue de 51 a 60 años, sexo masculino y con un nivel de instrucción superior, lo cual permite dar una valoración muy profesional al "CONEVI".

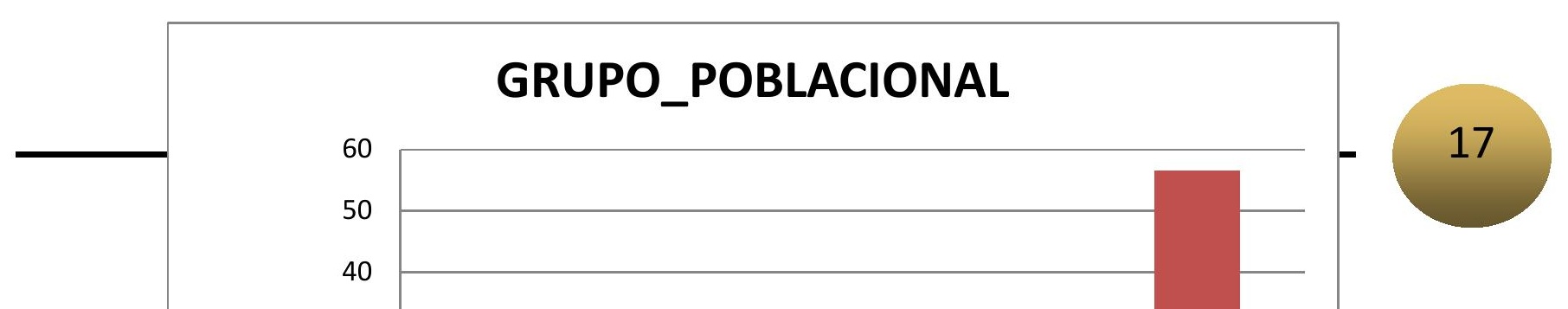


Gráfico 2. Grupo poblacional de la muestra de estudio

Fuente: Base de datos

Elaborado por: Autores

Interpretación: Se puede notar en el gráfico que existe variedad en la muestra al haber la presencia de personas con discapacidad, profesionales y personas sin discapacidad.

Tabla 2. Valoración del proyecto "CONEVI"

\begin{tabular}{|c|c|c|}
\hline PREGUNTA_1 & \begin{tabular}{|l} 
Frecuenci \\
a
\end{tabular} & $\begin{array}{l}\text { Porcenta } \\
\text { je }\end{array}$ \\
\hline $\begin{array}{l}\text { totalmente de } \\
\text { acuerdo } \\
\text { de acuerdo } \\
\text { ni de acuerdo ni en } \\
\text { desacuerdo } \\
\text { en desacuerdo } \\
\text { totalmente } \\
\text { desacuerdo en } \\
\text { total } \\
\end{array}$ & $\begin{array}{l}22 \\
8 \\
0 \\
0 \\
0 \\
30 \\
\end{array}$ & $\begin{array}{l}73,3 \\
26,7 \\
0 \\
0 \\
0 \\
100\end{array}$ \\
\hline PREGUNTA_2 & $\begin{array}{l}\text { Frecuenci } \\
\mathrm{a}\end{array}$ & $\begin{array}{l}\text { Porcenta } \\
\text { je }\end{array}$ \\
\hline $\begin{array}{l}\text { totalmente de } \\
\text { acuerdo } \\
\text { de acuerdo } \\
\text { ni de acuerdo ni en } \\
\text { desacuerdo } \\
\text { en desacuerdo }\end{array}$ & $\begin{array}{l}15 \\
11 \\
4 \\
0\end{array}$ & $\begin{array}{l}50 \\
36,7 \\
13,3 \\
0\end{array}$ \\
\hline
\end{tabular}




\begin{tabular}{|c|c|c|}
\hline $\begin{array}{l}\text { totalmente en } \\
\text { desacuerdo } \\
\text { Total }\end{array}$ & $\begin{array}{l}0 \\
30\end{array}$ & $\begin{array}{l}0 \\
100\end{array}$ \\
\hline PREGUNTA_3 & $\begin{array}{l}\text { Frecuenci } \\
\mathrm{a}\end{array}$ & $\begin{array}{l}\text { Porcenta } \\
\text { je }\end{array}$ \\
\hline $\begin{array}{l}\text { totalmente de } \\
\text { acuerdo } \\
\text { de acuerdo } \\
\text { ni de acuerdo ni en } \\
\text { desacuerdo } \\
\text { en desacuerdo } \\
\text { totalmente } \\
\text { desacuerdo } \\
\text { Total } \\
\end{array}$ & $\begin{array}{l}23 \\
5 \\
2 \\
0 \\
0 \\
30\end{array}$ & $\begin{array}{l}76,7 \\
16,7 \\
6,7 \\
0 \\
0 \\
100\end{array}$ \\
\hline PREGUNTA_4 & $\begin{array}{l}\text { Frecuenci } \\
\mathrm{a}\end{array}$ & $\begin{array}{l}\text { Porcenta } \\
\text { je }\end{array}$ \\
\hline $\begin{array}{l}\text { totalmente de } \\
\text { acuerdo } \\
\text { de acuerdo } \\
\text { ni de acuerdo ni en } \\
\text { desacuerdo } \\
\text { en desacuerdo } \\
\text { totalmente } \\
\text { desacuerdo } \\
\text { Total } \\
\end{array}$ & $\begin{array}{l}25 \\
4 \\
1 \\
0 \\
0 \\
30\end{array}$ & $\begin{array}{l}83,3 \\
13,3 \\
3,3 \\
0 \\
0 \\
100\end{array}$ \\
\hline PREGUNTA_5 & $\begin{array}{l}\text { Frecuenci } \\
\mathrm{a}\end{array}$ & $\begin{array}{l}\text { Porcenta } \\
\text { je }\end{array}$ \\
\hline $\begin{array}{l}\text { totalmente de } \\
\text { acuerdo } \\
\text { de acuerdo } \\
\text { ni de acuerdo ni en } \\
\text { desacuerdo } \\
\text { en desacuerdo } \\
\text { totalmente } \\
\text { desacuerdo } \\
\text { Total } \\
\end{array}$ & $\begin{array}{l}24 \\
5 \\
1 \\
0 \\
0 \\
30\end{array}$ & $\begin{array}{l}80 \\
16,7 \\
3,3 \\
0 \\
0 \\
100\end{array}$ \\
\hline
\end{tabular}

Fuente: Base de datos

Elaborado por: Autores 
Interpretación: En las preguntas de la encuesta hubo acuerdo total y unánime respecto a las instrucciones, manejo, respuesta del computador, seguridad del sistema y posibilidades de comunicación con la interfaz humano-computador.

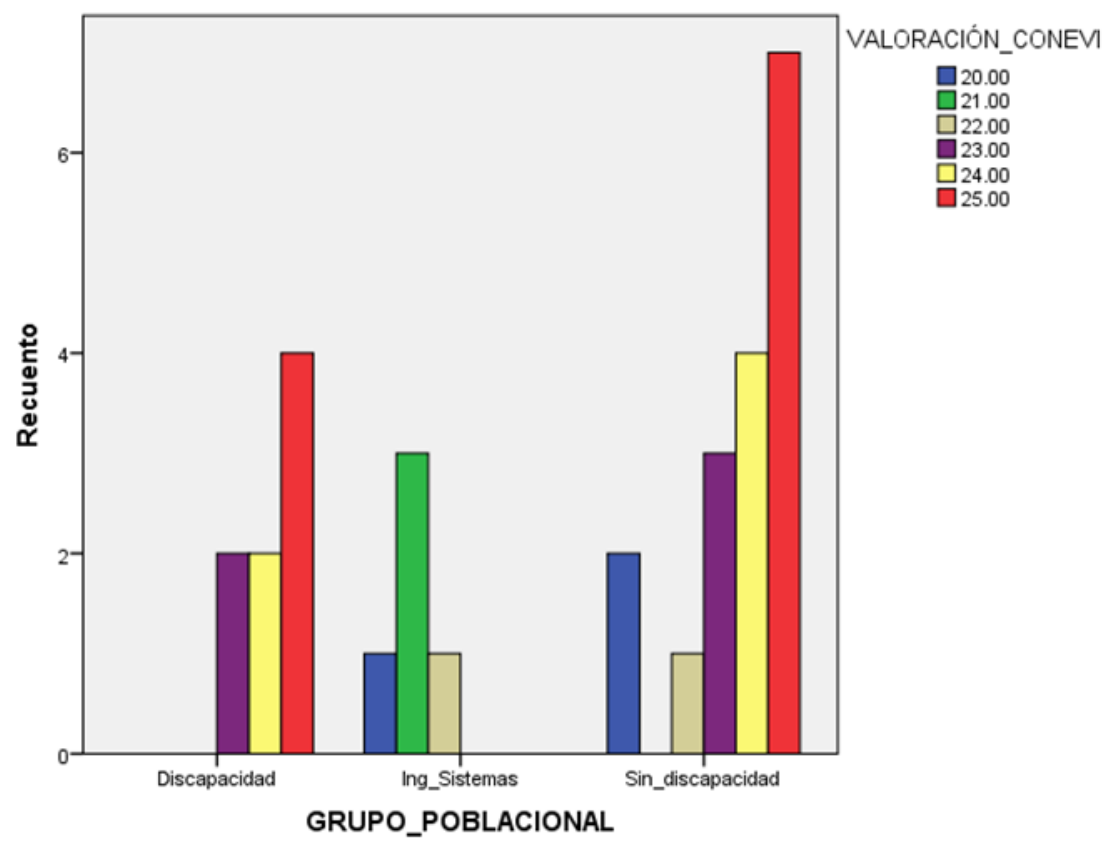

Gráfico 3. Grupo poblacional vs Valoración Conevi

Fuente: Base de datos

\section{Elaborado por: Autores}

Interpretación: Se puede apreciar que "Conevi" tiene una buena aceptación por parte de todos los usuarios tanto como profesionales, personas con discapacidad y personas sin discapacidad; ya que en la valoración del trabajo se obtuvo como mínimo un puntaje no menor a 20 puntos y además gran cantidad de los usuarios lo calificaron con la máxima puntuación de 25 puntos. 


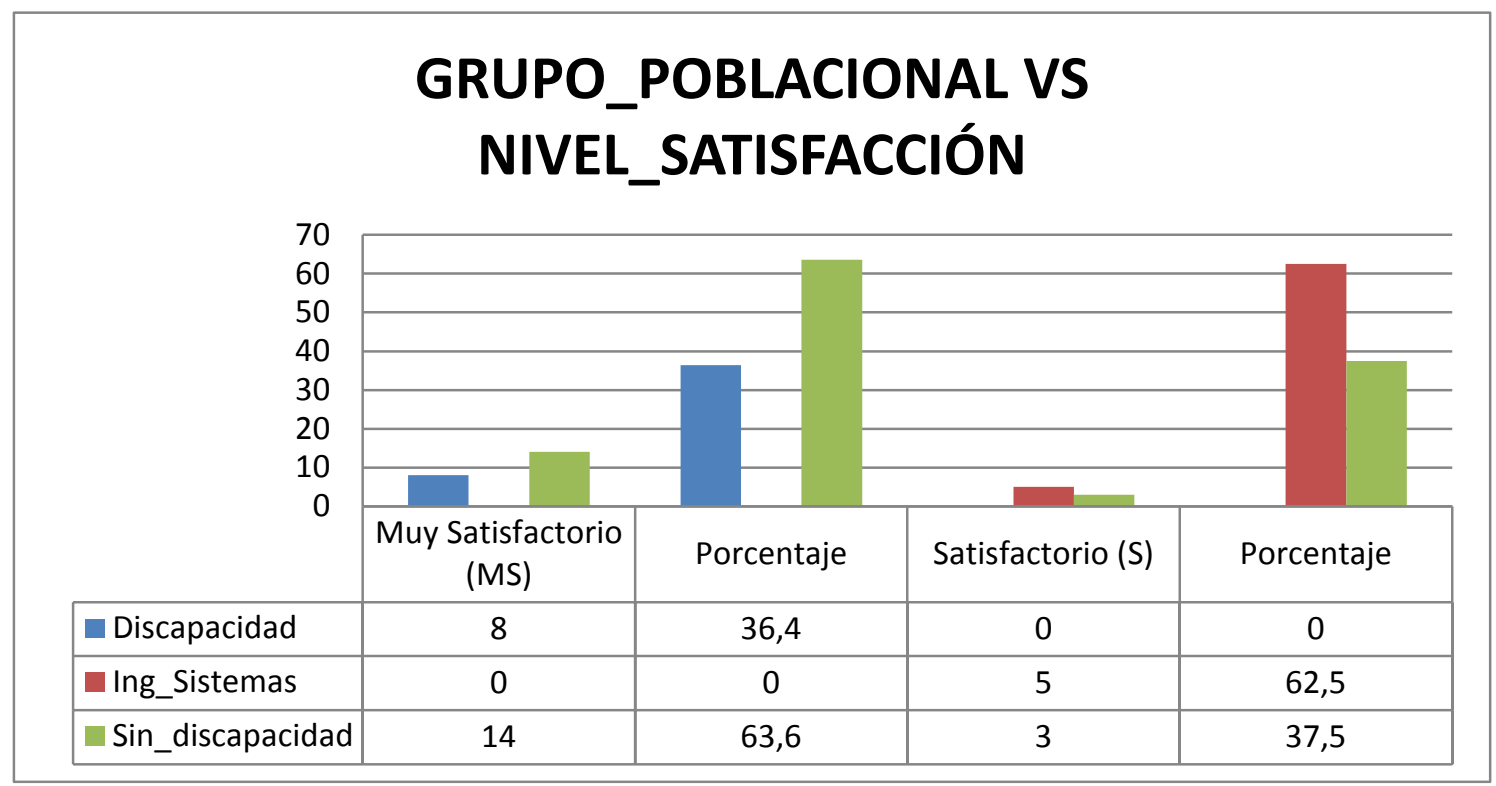

Gráfico 4. Grupo poblacional vs Nivel de satisfacción

Fuente: Base de datos

\section{Elaborado por: Autores}

Interpretación: En el Gráfico 4 podemos apreciar que el nivel de satisfacción ante la investigación que tuvieron las personas con discapacidad fue "Muy Satisfactorio (MS)", mientras que para los profesionales en Sistemas predominó el nivel "Satisfactorio" y para las personas sin discapacidad la mayoría también estuvo de acuerdo en que el resultado obtenido es "Muy Satisfactorio (MS)".

\section{Discusión.}

La interrelación estrecha del ser humano con la tecnología ha permitido desarrollar diferentes softwares basados en la programación, en la informática industrial y en el procesamiento digital de señales, los mismos que a través de algoritmos específicos utilizan las señales neuronales para el enlace y control de diversos dispositivos como los inalámbricos y computadores. Humanizando la tecnología, sus recursos han permitido derribar barreras comunicacionales que se pueden presentar en personas con alguna discapacidad motora (Koon \& de la Vega, 2014). 
Los resultados obtenidos en esta investigación tienen similitud con los resultados del trabajo desarrollado por Medina, Mejía y Alba (B. Medina, Mejía, \& Alba, 2017), quienes lograron que pacientes con tetraplejía controlaran el computador por medio de movimientos oculares. Así mismo Alagia reporta que tuvo características de funcionamiento parecidas al propuesto en este proyecto, ya que en su trabajo se da el uso de pestañeo voluntario para realizar acciones de control al ordenador (Alagia, 2018), al igual que él, Toapanta construyó una silla de ruedas eléctrica que mediante las ondas cerebrales al realizar parpadeos voluntarios, el usuario puede elegir la dirección en la que desea moverse con el fin de que personas con discapacidad puedan trasladarse de forma segura (Toapanta, 2018).

Altamirano y Revilla refieren que para la conversión $\mathrm{A} / \mathrm{D}$ y procesamiento de las señales se utilizó la tarjeta Arduino Uno, además de emplear el Bluetooth HC 05 - Arduino para una conexión inalámbrica y transferencia de los datos procesados (Altamirano \& Revilla, 2017), lo que difiere con este proyecto en el cual se realiza la conexión y toma de datos mediante el casco o diadema "Mindwave Mobile 2", y es coincidente con Calderón que mencionan el uso del dispositivo comercial "Mindwave Neurosky" para la captación de las diversas señales cerebrales existentes para su posterior uso en acciones de control de dispositivos externos (Calderón, 2016).

Para los proyectos referidos se utilizan en general dispositivos comerciales de EEG que permiten medir los ritmos cerebrales: Alfa, Beta, Gamma, Theta y Delta. Los cambios presentes en dichas señales según la actividad que la persona realice pueden ser usados para diversos propósitos, similar a este proyecto en el que se utiliza la variación en la señal Alfa para tareas de control en el ordenador, cabe recalcar que en los múltiples proyectos analizados la detección de rostros se realiza en el programa C++ con librerías Open CV (Ovejero, 2018), en esta investigación estas operaciones se realizan netamente en el programa "Matlab".

\section{Conclusiones.}

Al término de la investigación se concluye:

Que existen señales bioeléctricas cerebrales susceptibles de ser detectadas y traducidas por dispositivos electrónicos. 
Que estas señales bioeléctricas tienen un comportamiento específico de acuerdo a la actividad que el usuario realiza.

Que mediante algoritmos se pueden convertir estas señales en actividades sustitutas que el individuo no tiene la capacidad de realizar como el movimiento del cursor de un computador a través de la pupila.

Que la falta de iluminación adecuada en el entorno provoca fallas en la detección pupilar.

Que la valoración del algoritmo propuesto para cada etapa del proceso constató el correcto funcionamiento del sistema.

Que la evaluación del "CONEVI" evidencia su funcionamiento adecuado y utilidad infinita en la comunicación del usuario, computador y entorno.

\section{Bibliografia.}

Alagia, R. (2018). Procesamiento de artefactos en EEG para aplicaciones de comunicación y control. Recuperado de https://riunet.upv.es/handle/10251/104163

Altamirano, M., \& Revilla, E. (2017). Diseño y construcción del control de silla de ruedas motorizada basada en señales EEG para personas con severa discapacidad en el Hospital Regional de Lambayeque. Recuperado de http://repositorio.unprg.edu.pe/handle/UNPRG/1230

Alvarado, O., Tinoco, D., \& Veintimilla, J. (2016). Sistema Embebido para Detección de Somnolencia en Conductores mediante señal EEG. 10.

Calderón, D. (2016). Procesamiento de ondas cerebrales con microprocesador ARM para control de coche teledirigido. Recuperado de http://hdl.handle.net/11441/42446

Chang, A. (2014). Diseño y Simulación de un Robot Articular con seis grados de Libertad utilizando el Toolbox Robotics de Matlab para fortalecer las clases teóricas realizando prácticas de laboratorio con el software presentado en este proyecto. Recuperado de http://bibdigital.epn.edu.ec/handle/15000/8552

CONADIS. (s. f.). Estadísticas de Discapacidad. Recuperado 2 de febrero de 2019, de Tableau Software https://public.tableau.com/views/Discapacidad/Inicio?:embed=y\&:showVizHome=no\&:load OrderID=0\&:display_count=yes\&:showTabs=y

Ferreira, B., \& Rodríguez, C. (2017). Diseño de una interfaz gráfica en Matlab para controlar un sensor Cmos. JÓVENES EN LA CIENCIA, 2(1), 1023-1027. 
Giaconi, C., Pedrero, Z., \& San Martín, P. (2017). La discapacidad: Percepciones de cuidadores de niños, niñas y jóvenes en situación de discapacidad. Psicoperspectivas, 16(1), 55-67. https://doi.org/10.5027/psicoperspectivas-vol16-issue1-fulltext-822

Iglesias López, P. (2016). Brain-Computer Interfaces: Desarrollo de un sistema de identificación de estados mentales alfa (Info:eu-repo/semantics/bachelorThesis). Recuperado de http://oa.upm.es/39564/

Justo, F., \& Aguirre, I. (2014). Creación de una herramienta que permita mover el cursor de un computador a partir del movimiento ocular, utilizando técnicas de visión artificial. 7 Conferencia Latinoamérica y Caribe de Ingeniería y Tecnología. Recuperado de http://repositoriocdpd.net:8080/handle/123456789/388

Koon, R., \& de la Vega, M. (2014). El impacto tecnológico en las personas con discapacidad. 19.

Martín, J. (2015). Sistema Brain Computer Interface. Recuperado de https://upcommons.upc.edu/handle/2117/78057

Martín, M., \& Sánchez, M. (2016). Análisis cualitativo de tópicos vinculados a la calidad de vida en personas con discapacidad. Ciência \& Saúde Coletiva, 21, 2365-2374. https://doi.org/10.1590/1413-81232015218.04182016

Medina, B., Mejía, A., \& Alba, A. (2017). Sistema de Apoyo para Pacientes con Discapacidad Motriz Mediante una Interfaz Humano-Computadora Controlada por Movimientos Oculares. Memorias del Congreso Nacional de Ingeniería Biomédica, 3(1), 214-217.

Medina, L. (2018). Implementación de un prototipo de prótesis de mano usando el método bci no invasivo a través de estímulos endógenos. Recuperado de http://dspace.espoch.edu.ec/handle/123456789/9202

Moctezuma, L., Carrillo, M., Villaseñor, L., \& Torres, A. (2017). Hacia la clasificación de actividad e inactividad linguistica a partir de señales de electroencefalogramas (EEG). 15.

Morín, M., Santillán, A., Sainos, S., \& Oliveros, J. (2018). Prototipo de Silla de Ruedas Dirigida Usando Parpadeos. Revista Mexicana de Ingeniería Biomédica, 40(1), 1-13.

Ovejero, D. (2018). Detección del movimiento ocular para control de robots (Info:eurepo/semantics/bachelorThesis). Recuperado de http:/oa.upm.es/49701/

Pérez, I. (2017). Desarrollo de una plataforma para experimentación con Interface Cerebro Ordenador (BCI). Recuperado de http://repositorio.upct.es/handle/10317/6491

Puentes, A., Puentes, D., Puentes, E., \& Chávez, E. (2017). Fundamentos físicos de los procesos del organismo humano. Revista Cubana de Investigaciones Biomédicas, 36(2), 186-197. 
Comunicación parcial neurosensorial-visual a un ordenador mediante lectura de pupila

Rodríguez, J., \& Duque, N. (2016). Reconocimiento de expresiones faciales para interacción con el computador. JÓVENES EN LA CIENCIA, 2(1), 62-66.

Sánchez, J., Jofré, M., \& Burán, M. (2018). Las ondas alfa del electroencefalograma cuantificado y su relación con la evocación imaginaria de contenidos diferenciados. PSIUC REVISTA DE PSICOLOGÍA, 14.

Toapanta, C. (2018). Desarrollo de una silla de ruedas eléctrica controlado por ondas cerebrales usando el dispositivo Neurosky MindWave Mobile 2. Recuperado de https://repositorio.uisrael.edu.ec/handle/47000/1642 